\title{
Symmetries of Cauchy-Riemann Spaces
}

PAWEL NUROWSKI and JACEK TAFEL

Institute of Theoretical Physics, University of Warsaw, Warsaw. Poland

(Received: 11 September 1987)

Abstract. We simplify and generalize Cartan's results on Cauchy-Riemann spaces admitting continuous groups of automorphisms. We describe all such spaces in terms of local coordinates.

\section{Introduction}

Cauchy-Riemann spaces [1] appear in physics in the context of algebraically special solutions of the Einstein equations and null solutions of the Maxwell (or Yang-Mills) equations [2-4]. In both cases, spacetime admits a shearfree geodesic null congruence. This property is equivalent to the following condition on the metric tensor [5]

$$
g=\kappa \omega-p \alpha \bar{\alpha},
$$

where $\kappa$ (real) and $\alpha$ (complex) are one-forms on a three-dimensional submanifold $N$ of spacetime and

$$
\kappa \wedge \alpha \wedge \bar{\alpha} \neq 0 .
$$

The forms $\kappa$ and $\propto$ are defined by the congruence up to the transformations

$$
\begin{aligned}
& \kappa \rightarrow \varkappa^{\prime}=A \kappa, \quad A \neq 0, \\
& \alpha \rightarrow \alpha^{\prime}=B \alpha+C \kappa, \quad B \neq 0,
\end{aligned}
$$

where $A$ is a real function and $B, C$ are complex functions on $N$. A three-dimensional differential manifold $N$ with such a class of forms $(\kappa, \alpha)$ is called a Cauchy-Riemann space; it is also said to have a Cauchy-Riemann (CR) structure.

A CR structure on $N$ can be alternatively defined by means of a complex vector field $\partial$, such that $\hat{\partial}$ and $\bar{\partial}$ are independent at each point of $N$. A mutual relation between $\partial$ and $(\kappa, \alpha)$ is established by the requirement that $(\kappa, \alpha, \bar{\alpha})$ is a basis dual to $\left(\partial_{0}, \partial, \bar{\partial}\right)$, where $\partial_{0}$ is a real vector field. The replacement (3) implies the following 'gauge' transformation of $\partial$

$$
\hat{\theta} \rightarrow \partial^{\prime}=B^{-1} \partial
$$

If $\kappa$ and $\alpha$ (or $\partial$ ) are sufficiently regular (e.g., real analytic) then the CR space can be identified (locally) with a real hypersurface in $\mathbb{C}^{2}$ (see, e.g., [4]). This hypersurface is given parametrically by

$$
\left(\xi\left(x^{i}\right), \eta\left(x^{i}\right)\right) \in \mathbb{C}^{2},
$$


where $x^{i}, i=1,2,3$, are coordinates of $N$ and $\xi$ and $\eta$ are two solutions of the equation

$$
\mathrm{d} f \wedge \kappa \wedge \alpha=0
$$

satisfying the independence condition

$$
\mathrm{d} \xi \wedge \mathrm{d} \bar{\xi} \wedge \mathrm{d} \eta \neq 0 \text {. }
$$

Conversely, given a hypersurface $\Sigma$ in $\mathbb{C}^{2}$ defined by

$$
R(\xi, \eta, \bar{\zeta}, \bar{\eta})=0,
$$

where $R$ is a real function such that $R_{\eta} \neq 0$ on $\Sigma$, we can define the CR structure on $\Sigma$ by taking

$$
\kappa=i\left(R_{\xi} \mathrm{d} \xi+R_{\eta} \mathrm{d} \eta\right), \quad \alpha=\mathrm{d} \xi .
$$

In this Letter we study the local properties of CR structures admitting infinitesimal symmetries. Basic results in this field were obtained by Elie Cartan [6], who found all hypersurfaces in $\mathbb{C}^{2}$ invariant (globally or locally) with respect to a transitive group of biholomorphic transformations of $\mathbb{C}^{2}$. We present here another approach, which leads directly to the forms $\kappa$ and $\alpha$ expressed in terms of 'canonical' coordinates. We consider the case of nontransitive actions (Sections 3 and 4) and transitive ones (Section 5).

With the exception of Section 2, we shall assume that CR structures under investigation are nonintegrable, i.e.,

$$
\kappa \wedge \mathrm{d} \kappa \neq 0
$$

or, equivalently,

$$
\partial, \bar{\partial},[\partial, \bar{\partial}] \text { are independent at each point. }
$$

Such structures correspond to twisting congruences in spacetime [7].

Our considerations are purely local. We assume that all manifolds and fields are of class $C^{\infty}$. Incidentally, equations of the Lewy type $[8,5]$ do not occur when a CR space admits symmetries.

\section{Groups of Automorphisms}

A diffeomorphism $\phi: N \rightarrow N$ is called a symmetry (automorphism) of a Cauchy-Riemann space iff the pullbacks $\phi^{*} \kappa, \phi^{*} \alpha$ are related to $\kappa, \alpha$ by the transformation (3) [4]. An equivalent condition is $\phi_{*} \partial \sim \partial$. We say that a vector field $X$ is an infinitesimal symmetry if

$$
\mathscr{L}_{x} \kappa=a \kappa, \quad \mathscr{L}_{x} \alpha=b \alpha+c \kappa,
$$

or, equivalently,

$$
[X, \partial]=-b \partial,
$$

where $\mathscr{L}_{x}$ denotes the Lie derivative along $X, a$ is a real function, and $b, c$ are complex functions. 
If a Cauchy-Riemann structure is integrable, then $\kappa \wedge \mathrm{d} \kappa=0$ and it follows from the Frobenius theorem that $\kappa \sim \mathrm{d} u$, where $u$ is a real function. Then Equation (5) admits a complex solution $\xi$, hence $\alpha=B \mathrm{~d} \xi+C k$. By virtue of transformations (3), we can assume

$$
\kappa=\mathrm{d} u, \quad \alpha=\mathrm{d} \xi
$$

without loss of generality. If $\eta=u$ then the hypersurface in $\mathbb{C}^{2}$ given by (4) is the plane $\operatorname{Im} \eta=0$.

In this case the local symmetry group is infinitely dimensional. It consists of transformations

$$
u \rightarrow f(u), \quad \xi \rightarrow h(\xi, u) .
$$

If a Cauchy-Riemann structure is nonintegrable, then it follows from a remark of Segre that its symmetry group is a finite-dimensional Lie transformation group [6]. In virtue of the Palais theorem [9], this fact can be considered as a direct consequence of Equations (11) and their integrability conditions. They show that the exterior derivatives of $\partial_{0} a, b, c$ and components of $X$ must be linear functions of these variables with coefficients defined by $k$ and $\alpha$. Hence, given a CR structure, a general solution $X$ of the symmetry conditions (11) depends at most on 8 real parameters (values of $\tilde{\sigma}_{0} a, b, c$ and components of $X$ at a fixed point, provided no further constraints follow). Thus, the Lie algebra generated by infinitesimal symmetries has dimension $D \leqslant 8$. The Palais theorem says that the corresponding group of transformations is a Lie transformation group of dimension $D \leqslant 8$.

In the following, we investigate separately the cases when $D$ is equal to 1,2 or $D \geqslant 3$.

\section{3. $D=1$}

If only one infinitesimal symmetry is present, then we can easily adjust a transformation (3) in order to get

$$
\mathscr{L}_{x} \kappa=0, \quad \mathscr{L}_{x} \alpha=0
$$

It follows from (15) that $\kappa=A_{i}(x, y) \mathrm{d} x^{i}$ and $\alpha=B_{i}(x, y) \mathrm{d} x^{\varepsilon}$ in coordinates $x^{t}=(u, x, y)$ such that $X=\hat{\partial}_{u}$. Condition (9) requires $A_{1} \neq 0$. A residual freedom of transformations (3) allows us to assume $A_{1}=1$ and $B_{1}=0$. Then $\alpha$ becomes proportional to an exact form $\mathrm{d} \xi$, where $\xi=\xi(x, y)$. The functions $\operatorname{Re} \xi$, Im $\xi$ can be chosen as new coordinates $x, y$. A suitable transformation of $\alpha$ and $u$ leads to the following canonical expressions for $\kappa$ and $\alpha$

$$
\kappa=\mathrm{d} u+f(x, y) \mathrm{d} x, \quad \alpha=\mathrm{d} x+i \mathrm{~d} y,
$$

where $\partial_{y} f \neq 0$. Particular solutions $\xi$ and $\eta$ of (5) are given by

$$
\xi=x+i y, \quad \eta=u+h(x, y),
$$


where $2 h_{\bar{\xi}}=f$. It follows from (17) that

$$
\operatorname{Im} \eta=F(\operatorname{Re} \xi, \operatorname{Im} \xi),
$$

where $F=\operatorname{Im} h$ and $F_{\zeta \bar{\xi}} \neq 0$.

THEOREM 1. If a CR structure admits a one-dimensional group of symmetries then it is equivalent to the $C R$ structure defined by (16) with some $f$. The corresponding hypersurface in $\mathbb{C}^{2}$ is given by Equation (18).

\section{4. $D=2$}

It is easy to show that the orbits of a two-dimensional symetry group $G$ must be two-dimensional. Let us assume that it is not so. Then the vector fields $X_{1}$ and $X_{2}$ representing any two independent infinitesimal symmetries have to be proportional at each point, i.e., $X_{1}=q X_{2}$, where $q \neq$ const (otherwise $X_{1}$ and $X_{2}$ represent the same symmetry). It follows from (12) that either $\partial \sim X_{2}$ or $\partial q=0$. The first possibility is in contradiction with (10) and the second violates (10) or the assumption $q \neq$ const (since $\partial q=0$ implies $\bar{\partial} q=0$ and $[\partial, \bar{\partial}] q=0$ ).

Since $X_{r}, r=1,2$, are infinitesimal symmetries we know that

$$
\mathscr{L}_{r} \kappa=a_{r} \kappa, \quad \mathscr{L}_{r} \alpha=b_{r} \alpha+c_{r} \kappa,
$$

where $\mathscr{L}_{r}$ denotes the Lie derivative along $X_{r}$. We now want to transform $\kappa$ and $\alpha$ to forms $\kappa^{\prime}, \alpha^{\prime}$ strictly invariant under the action of $G$,

$$
\mathscr{L}_{r} \kappa^{\prime}=0, \quad \mathscr{L}_{r} \alpha^{\prime}=0
$$

Comparing (19) and (20) yields the following equations for the parameters $A, B, C$ of the required transformation (3)

$$
\mathscr{L}_{r} A=-A a_{r}, \quad \mathscr{L}_{r} B=-B b_{r}, \quad \mathscr{L}_{r} C=-C a_{r}-B c_{r}
$$

Solutions to these equations exist since the integrability conditions of (21) follow from equations obtained by the Lie differentiation of (19). Hence, we can assume (20) without loss of generality.

There are two nonisomorphic two-dimensional Lie algebras. We can choose the fields $X_{r}$ in such a way that

$$
\left[X_{1}, X_{2}\right]=\varepsilon X_{1}, \varepsilon=0,1 .
$$

Since the orbits of $G$ are two-dimensional, it follows from (22) that

$$
X_{1}=\partial_{u}, \quad X_{2}=\varepsilon u \partial_{u}+\partial_{x}
$$

in some coordinates $u, x, y$. Now it is easy to find general forms $\kappa^{\prime}$ and $\alpha^{\prime}$ satisfying Equations (20). By virtue of residual transformations (3), and a freedom in the choice of the coordinates, they can be reduced to the following expressions

$$
\kappa=\exp (-\varepsilon x) \mathrm{d} u+f(y) \mathrm{d} x, \quad \alpha=\mathrm{d} x+i \mathrm{~d} y,
$$


where $\partial_{y} f \neq 0$ and we have dropped the primes. Particular solutions $\xi$ and $\eta$ of Equation (5) are given by

$$
\xi=x+i y, \quad \eta=u+h(y) \exp (\varepsilon x)
$$

where $i h_{y}+\varepsilon h=f$. It follows from (25) that

$$
\operatorname{Im} \eta=\exp (\varepsilon \operatorname{Re} \xi) F(\operatorname{Im} \xi),
$$

where $F=\operatorname{Im} h$. By an appropriate choice of $f$ we can obtain any real function $F$ satisfying the condition $F_{y y}+\varepsilon F \neq 0$.

THEOREM 2. If a CR structure admits a two-dimensional group of symmetries, then it is equivalent to the structure defuned by (24). The corresponding hypersurface in $\mathbb{C}^{2}$ is given by Equation (26).

\section{5. $D \geqslant 3$}

Our approach to this case can be described as follows. First we observe that any Lie group of dimension $D \geqslant 3$ contains locally a three-dimensional subgroup. Hence, in order to find all symmetrical CR structures with $D \geqslant 3$, it is sufficient to consider the case $D=3$. We prove that the action of a three-dimensional symmetry group $G$ on $N$ is (locally) simply transitive and the forms $\kappa$ and $\alpha$ can be transformed to left invariant forms on $G$. In virtue of the Bianchi classification of three-dimensional Lie groups the whole problem reduces to the problem of finding all nonequivalent left invariant frames on these groups.

LEMMA. Any Lie algebra $\mathscr{G}^{\prime}$ of dimension greater then two contains a three-dimensional subalgebra $\mathscr{G}$

Proof. According to the Levi-Malcev theorem [10], $\mathscr{G}$ can be decomposed into the semidirect sum of a solvable subalgebra $\mathscr{R}$ (radical) and a semisimple subalgebra $\mathscr{S}$. If $\mathscr{F}$ is nontrivial, then it contains either the subalgebra su(2) (if $\mathscr{F}$ is compact [11, p. 219]) or $\operatorname{su}(1,1)$ (if $\mathscr{P}$ is noncompact $[11$, p. 245]). If $\mathscr{P}$ is trivial then $\operatorname{dim} \mathscr{R} \geqslant 3$ and $\mathscr{R}$ must contain a three-dimensional subalgebra since any solvable algebra $\mathscr{R}$ contains subalgebras of all dimensions between 1 and $\operatorname{dim} \mathscr{R}[11, \mathrm{p} .133]$.

The lemma has its local equivalent at the group level. Hence, it follows that highly symmetrical $(D \geqslant 4)$ CR structures are particular cases of CR structures with a threedimensional symmetry of group $G$.

It is easy to prove that orbits of $G$ are three-dimensional. It follows already from Section 4 that they cannot be one-dimensional. Let us assume for a moment that they are two-dimensional, i.e.,

$$
X_{3}=p X_{1}+r X_{2}, \quad X_{2} \neq q X_{1}
$$

where $X_{r}(r=1,2,3)$ are independent infinitesimal symmetries and either $p \neq$ const or 
$r \neq$ const. It follows from (12) that

$$
\left(\partial p X_{1}+\partial r X_{2}\right) \sim \partial .
$$

Equation (28) and its conjugate yield

$$
X_{r}=s_{r} \partial+\bar{s}_{r} \bar{\partial},
$$

where $s_{r}$ are complex nonvanishing functions. Substituting (29) into (12) yields contradiction with (10).

Since the orbits are three-dimensional the manifold $N$ can be locally identified with the group manifold $G$ and the action of $G$ on $N$ can be identified with the natural left action of $G$ on itself.

An analysis analogous to that for $D=2$ shows that the forms $\kappa$ and $\alpha$ satisfying the symmetry conditions (19) (now $r=1,2,3$ ) can be replaced by new forms, which satisfy the equations

$$
\mathscr{L}_{r} \kappa=0, \quad \mathscr{L}_{r} \alpha=0 .
$$

It follows from (30), (2), (9) and the identification of $N$ with $G$ that $(\kappa, \operatorname{Re} \alpha, \operatorname{Im} \alpha)$ is a left-invariant frame on $G$ satisfying (9).

THEOREM 3. If a CR structure admits a symmetry group $G^{\prime}$ of dimension $D \geqslant 3$ then it is equivalent to the $C R$ structure defined by a left-invariant basis $(\kappa, \operatorname{Re} \alpha, \operatorname{Im} \alpha)$, $\kappa \wedge \mathrm{d} \kappa \neq 0$, on a three-dimensional local subgroup $G$ of $G^{\prime}$.

The local properties of $G$ are determined by its Lie algebra $\mathscr{G}$. All the threedimensional Lie algebras are explicitly known (see, e.g., [12]). Let $\left(\vartheta^{r}\right)$ be a particular left invariant basis related to $\mathscr{G}$. Then $\kappa$ and $\alpha$ are general linear combinations of $\theta^{r}$ with constant coefficients such that (9) is satisfied. A number of free parameters can be reduced by means of transformations (3) (with constant $A, B, C$ ) and transformations of $\xi^{r}$ preserving the Maurer-Cartan equations. We can always obtain $\alpha \wedge \mathrm{d} \alpha=0$, hence $\alpha \sim \mathrm{d} \xi$ and $\xi$ satisfies Equation (5). If $\mathscr{G}=\operatorname{su}(2)$ or $\operatorname{su}(1,1)$ (types IX and VIII), then one real parameter remains. It means that one-parameter families of CR structures are related to these algebras. For other algebras (except I and $\mathrm{V}$, which are excluded by (9)), there is only one corresponding CR structure. For all Bianchi types (except I and V) we list below the following data

(i) reduced forms $\kappa$ and $\alpha$ in terms of coordinates $u, \xi, \bar{\xi}$ or $u, x, y$ where $\xi=x+i y$ and $u, x, y$ are real,

(ii) symmetry transformations generated by $G$ ( $p, q, r$ being constant parameters),

(iii) a second solution $\eta$ of (5) and an equation of the hypersurface in $\mathbb{C}^{2}$ defined by (4).

We also give the corresponding Cartan type [6] in square brackets.

Type II [A]

(i) $x=\mathrm{d} u-i \bar{\xi} \mathrm{d} \xi+i \xi \mathrm{d} \bar{\xi}, \quad \alpha=\mathrm{d} \xi$, 
(ii) $u^{\prime}=u+i \bar{q} \xi-i q \bar{\zeta}+p, \quad \xi^{\prime}=\xi+q, \quad q \in \mathbb{C}, p \in R$,

(iii) $\eta=u+i \xi \bar{\xi}, \quad \operatorname{Im} \eta=\xi \bar{\xi}$.

Type IV [F]

(i) $\kappa=y^{-1}(\mathrm{~d} u-\ln y \mathrm{~d} x), \quad \alpha=y^{-5}(\mathrm{~d} x+i \mathrm{~d} y)$,

(ii) $u^{\prime}=r u+r x \ln r y+p, \quad x^{\prime}=r x, \quad y^{\prime}=r y+q, \quad p, q, r \in R, \quad r>0$,

(iii) $\eta=u+x+i y \ln y, \quad \operatorname{Im} \eta=\operatorname{Im} \xi \ln (\operatorname{Im} \xi)$.

Type $\mathrm{VI}_{h}$ (including $\mathrm{VI}_{0}$ and $\mathrm{III}$ ) [E, B]

(i) $k=y^{b} \mathrm{~d} u-y^{-1} \mathrm{~d} x, \quad \alpha=y^{-1}(\mathrm{~d} x+i \mathrm{~d} y), \quad$ where $b=\frac{1-\sqrt{-h}}{1+\sqrt{-h}}$,

(ii) $u^{\prime}=r^{-b} u+p, \quad x^{\prime}=r x, \quad y^{\prime}=r y+q, q, q, r \in R, r>0$,

(iii) $\eta=-b u+i y^{-b}, \quad \operatorname{Im} \eta=(\operatorname{Im} \xi)^{-b}$, for $h \neq 1$,

$-\eta=u+i \ln y, \quad \operatorname{Im} \eta=(\operatorname{Im} \xi), \quad$ for $h=-1$ (type III).

Type VII $_{h}$ (including $\mathrm{VII}_{0}$ ) [H]

(i) $k=\mathrm{d} u+\mathrm{e}^{(A+i) u} \mathrm{~d} \xi+\mathrm{e}^{(A-i) u} \mathrm{~d} \bar{\xi}, \quad \alpha=\mathrm{e}^{(A+i) u} \mathrm{~d} \xi$, where $A=\sqrt{h}$,

(ii) $u^{\prime}=u-p, \quad \xi^{\prime}=\mathrm{e}^{(A+i) p} \xi+q, \quad p \in R, q \in \mathbb{C}$,

(iii) $\eta=(i-A) \bar{\xi}+\mathrm{e}^{(i-A)_{u}}, \quad \operatorname{Im}[\eta+(A-i) \bar{\xi}]^{A+i}=0$.

Type IX $[\mathrm{D}, \mathrm{L}]$ (upper signs) and VIII [C, K] (lower signs)

(i) $k=\mathrm{d} u+\frac{k \mathrm{e}^{i t u}-i \bar{\xi}}{\xi \bar{\xi} \pm 1} \mathrm{~d} \xi+\frac{k \mathrm{e}^{-i u}+i \xi}{\xi \bar{\xi} \pm 1} \mathrm{~d} \bar{\xi}, \quad \alpha=\frac{2 \mathrm{e}^{i t}}{\xi \bar{\xi} \pm 1} \mathrm{~d} \xi$,

where $0 \leqslant k \in R, k^{2} \pm 1 \neq 0$,

(ii) $u^{r}=u-i \ln (\bar{q} \xi+\bar{p})+i \ln (q \bar{\xi}+p), \quad \xi^{i}=\frac{p \xi \mp q}{\bar{q} \bar{\xi}+\bar{p}}$

where $p \bar{p} \pm q \bar{q}=1$ and $p, q \in \mathbb{C}$,

(iii) $\eta=\frac{\xi \mathrm{e}^{i u}-i k}{\mathrm{e}^{i u} \pm i k \bar{\xi}}, \quad|\xi-\eta|=k|1 \pm \xi \bar{\eta}|$, for $k>0$,

$\eta=\left[(\xi \bar{\zeta} \pm 1) \mathrm{e}^{-m}\right]^{1 / 2}, \quad \eta \bar{\eta}=|\xi \bar{\xi} \pm 1|, \quad$ for $k=0$.

\section{Concluding Remarks}

As we have seen in Section 2, all integrable Cauchy-Riemann structures are isomorphic and admit locally an infinitely dimensional group of automorphisms. A nonintegrable 
structure may have no symmetries. If it does, then they form a Lie transformation group of dimension $D \leqslant 8$. The action of this group is transitive if $D \geqslant 3 . \mathrm{CR}$ structures with continuous symmetries $(D \geqslant 1)$ are locally equivalent to the structures defined by (16), (24) or the forms $\kappa, \alpha$ listed in Section 5 . We have found the corresponding hypersurfaces in $\mathbb{C}^{2}$. Those for $D \geqslant 3$ are locally equivalent to the hypersurfaces obtained by Cartan [6].

Finally we make a comment on CR structures admitting groups of symmetries of dimension $D \geqslant 4$. It was proved by Cartan [6] that, from a local point of view, they are all isomorphic and admit the group $\mathrm{SU}(1,2)$. They can be characterized by the vanishing of the Cartan relative invariant $r$ [6, Ch. III]. They are known to physicists since they are related to the Robinson congruence [13,3], which exists, e.g., in Minkowski space. On our list in Section 5, these structures occur for the types II, III, VI ${ }_{-9}$, VIII $(k=0$ or $k=\sqrt{2})$, IX $(k=0)$. The other structures from this list are nonisomorphic and admit precisely three-dimensional groups of automorphisms.

\section{Acknowledgements}

We are grateful to Prof. A. Trautman for encouragement and critical reading of the manuscript. We also acknowledge helpful discussions with J. Lewandowski, Prof. I. Robinson, and Dr A. Strassburger. This work was partially supported by the Polish Research Programme CPBP 01.03.

\section{References}

1. Wells, Jr, R. O., Function theory on differentiable manifolds, in Contributions to Analysis, Academic Press, New York, 1974.

2. Penrose, R. and Rindler, W., Spinors and Spacetime, vol. 2, Cambridge University Press, Cambridge, 1986.

3. Robinson, I. and Trautman, A, Cauchy-Riemann structures in optical geometry, in R. Rufini (ed.), Proc. Fourth Marcel Grossman Meeting on General Reiativity, Elsevier, Amsterdam, 1986.

4. Robinson, I. and Trautman, A., Optical geometry, Warsaw University preprint IFT/7/87 (1987).

5. Tafel, J., Leti. Math. Phys. 10, 33 (1985).

6. Cartan, E., Ann. Math. Pura Appl. 11, 17 (1932).

7. Robinson, I. and Trautman, A., J. Math. Phys. 24, 1425 (1983).

8. Jacobowitz, H. and Treves, F., Invent. Math. 66, 231 (1982).

9. Kobayashi, S., Transformation Groups in Differential Geometry, Springer-Verlag, Berlin, 1972.

10. Barut, A. and Rackka, R., Theory of Group Representations and Applications, PWN-Polish Scientific Publishers, Warsaw, 1977.

11. Helgason, S., Differential Geometry and Symmetric Spaces, Academic Press, New York and London, 1962.

12. Kramer, D., Stephani, H., MacCallum, M., and Herlt, H., Exact Sotutions of Einstein's Field Equations, VEB Deutscher Verlag der Wissenschaften, Berlin, 1980.

13. Penrose, R., J, Math. Phys, 8, 345 (1967). 